\title{
EXTREME POINTS IN A CLASS OF POLYNOMIALS HAVING UNIVALENT SEQUENTIAL LIMITS
}

\author{
BY \\ T. J. SUFFRIDGE $\left({ }^{1}\right)$
}

\begin{abstract}
This paper concerns a class $\mathscr{P}_{n}$ (defined below) of polynomials of degree less than or equal to $n$ having the properties: each polynomial which is univalent in the unit disk and of degree $n$ or less is in $\mathscr{P}_{n}$ and if $\left\{P_{n_{k}}\right\}_{k=1}^{\infty}$ is a sequence of polynomials such that $P_{n_{k}} \in \mathscr{P}_{n_{k}}$ and $\lim _{k \rightarrow \infty} P_{n_{k}}=f$ (uniformly on compact subsets of the unit disk) then $f$ is univalent. The approach is to study the extreme points in $\mathscr{P}_{n}\left(P \in \mathscr{P}_{n}\right.$ is extreme if $P$ is not a proper convex combination of two distinct elements of $\mathscr{P}_{n}$ ). Theorem 3 shows that if $P \in \mathscr{P}_{n}$ is extreme then $((n+1) / n) P(z)-(1 / n) z P^{\prime}(z)$ is univalent and Theorem 6 gives a geometric condition on the image of the boundary of the disk under this mapping in order that $P$ be extreme. Theorem 10 states that the collection of polynomials univalent in the unit disk and having the property $P(z)=z+a_{2} z^{2}+\cdots$ $+a_{n} z^{n}, a_{n}=1 / n$, are dense in the class $S$ of normalized univalent functions. These polynomials have the very striking geometric property that the tangent line to the curve $P\left(e^{i \theta}\right), 0 \leqq \theta \leqq 2 \pi$, turns at a constant rate (between cusps) as $\theta$ varies.
\end{abstract}

For $n \geqq 1$, let $\mathscr{P}_{n}$ be the collection of polynomials of degree less than or equal to $n$ of the form $P(z)=z+a_{2} z^{2}+\cdots+a_{n} z^{n}$ such that the equations

$$
\begin{aligned}
\frac{\Delta_{k} P(z)}{z} & =\frac{P\left(z e^{i k \pi /(n+1)}\right)-P\left(z e^{-i k \pi /(n+1)}\right)}{z\left(e^{i k \pi /(n+1)}-e^{-i k \pi /(n+1)}\right)} \\
& =1+\sum_{j=2}^{n} a_{j} \frac{\sin k j \pi /(n+1)}{\sin k \pi /(n+1)} z^{j-1}=0, \quad k=1,2, \ldots, n,
\end{aligned}
$$

have no roots in $|z|<1$. Since $P$ is univalent in $|z|<1$ if and only if for $0<\theta<\pi / 2$ the equation

$$
O=1+\sum_{j=2}^{n} a_{j} \frac{\sin j \theta}{\sin \theta} z^{j-1}
$$

has no roots in $|z|<1$, [3], $\mathscr{P}_{n}$ contains the collection $U_{n}$ of all univalent polynomials of degree $n$ or less which are appropriately normalized.

We say that $P \in \mathscr{P}_{n}$ is an extreme point of $\mathscr{P}_{n}$ if there do not exist $P_{1}$ and $P_{2}$ in $\mathscr{P}_{n}$, $P_{1} \neq P_{2}$, such that $P=t P_{1}+(1-t) P_{2}$ where $0<t<1$. We will show below that $\bigcup_{n=1}^{\infty} \mathscr{P}_{n}$ is a normal family and it is then easy to see that, for each $n, \mathscr{P}_{n}$ is a compact

Presented to the Society, January 22, 1971; received by the editors October 2, 1970 and, in revised form, March 1, 1971.

AMS 1969 subject classifications. Primary 3010, 3042, 3043.

Key words and phrases. Extreme point, convex hull, univalent polynomial.

(1) This work was supported by the National Science Foundation under grant number GP 19533.

Copyright (C) 1972, American Mathematical Society 
subset of a locally convex linear topological space. As in [2], the Krein-Milman theorem [4] then applies and $\mathscr{P}_{n}$ is contained in the closure of the convex hull of its extreme points. Further, any continuous linear functional on $\mathscr{P}_{n}$ assumes its maximum real part and maximum modulus on the set of extreme points.

It is clear that $P \in \mathscr{P}_{n}$ is an extreme point if and only if for each real $\alpha, e^{-i \alpha} P\left(z e^{i \alpha}\right)$ is extreme. Hence in attempting to characterize the extreme points of $\mathscr{P}_{n}$ we may assume $P(z)=z+a_{2} z^{2}+\cdots+a_{n} z^{n}$ where $a_{n} \geqq 0$.

THEOREM 1. If $P(z)=z+\sum_{j=2}^{n} a_{j} z^{j}$ is an extreme point of $\mathscr{P}_{n}$ such that $a_{n} \geqq 0$ then $a_{j+1}=\bar{a}_{n-j}, 0 \leqq j \leqq n-1$.

Proof. Note that

$$
\begin{aligned}
\sin k(j+1) \pi /(n+1) & =(-1)^{k-1} \sin [k \pi-k(j+1) \pi /(n+1)] \\
& =(-1)^{k-1} \sin k(n-j) \pi /(n+1)
\end{aligned}
$$

so that $a_{n} \leqq 1$ with equality only if all the roots of the equations $\Delta_{k} P(z) / z=0$ lie on $|z|=1$ and in this case $a_{j+1}=\bar{a}_{n-j}$ [1]. Thus we need only show $a_{n}=1$.

Let

$$
\hat{P}(z)=z^{n+1} \overline{P(1 / \bar{z})}=\sum_{j=1}^{n} \bar{a}_{n-j+1} z^{j}
$$

and observe that $\Delta_{k}(\hat{P})=(-1)^{k-1}\left(\Delta_{k} P\right)^{\wedge}$. Assume $1>a_{n}$ and define $Q(z)$ $=\left(1+a_{n}\right)^{-1}[P(z)+\hat{P}(z)], R(z)=\left(1-a_{n}\right)^{-1}[P(z)-\hat{P}(z)]$. We now show $Q, R \in \mathscr{P}_{n}$. For any polynomial $S$ of degree less than or equal to $n$,

$$
\hat{S}\left(e^{i \theta}\right)=e^{i(n+1) \theta} \overline{S\left(e^{i \theta}\right)}
$$

so $\Delta_{k} \hat{P} / \Delta_{k} P=(-1)^{k-1}\left(\Delta_{k} P\right)^{\wedge} / \Delta_{k} P$ is analytic in a neighborhood of the closed disk. But

$$
\begin{aligned}
\left|\Delta_{k} \hat{P}(z) / \Delta_{k} P(z)\right| & =1 & & \text { on }|z|=1, \\
& =a_{n} & & \text { at } z=0,
\end{aligned}
$$

so $\left|\Delta_{k} \hat{P}\right| \Delta_{k} P \mid<1$ in $|z|<1$. This means $\Delta_{k} Q \neq 0 \neq \Delta_{k} R$ in $0<|z|<1$ so $Q, R \in \mathscr{P}_{n}$. However $P=\left(1+a_{n}\right) / 2 Q+\left(1-a_{n}\right) / 2 R$ and $P$ is extreme so $Q=R$. This implies $\hat{P}=a_{n} P$ and equating $n$th coefficients, $a_{n}^{2}=1, a_{n}=1$ which is a contradiction. This completes the proof of Theorem 1 .

Now consider the polynomials

$$
Q_{p}(z ; n)=\sum_{j=1}^{n} \frac{\sin j p \pi /(n+1)}{\sin p \pi /(n+1)} z^{j}=\frac{z\left(1-(-1)^{p} z^{n+1}\right)}{1-2 z \cos p \pi /(n+1)+z^{2}}, \quad 1 \leqq p \leqq n .
$$

Since

(3) $\begin{aligned} & \Delta_{k} Q_{p}(z ; n) \\ & =\frac{z\left(1-(-1)^{k+p} z^{n+1}\right)\left(1-z^{2}\right)}{\left(1-2 z e^{i k \pi /(n+1)} \cos p \pi /(n+1)+z^{2} e^{2 i k \pi /(n+1)}\right)\left(1-2 z e^{-i k \pi /(n+1)} \cos p \pi /(n+1)+z^{2} e^{-24 k \pi /(n+1)}\right)}\end{aligned}$, 
$1 \leqq k \leqq n, 1 \leqq p \leqq n$, each have $n-1$ zeros on $|z|=1$, we conclude $Q_{p}(z ; n) \in \mathscr{P}_{n}$. Also, we see that

$$
\begin{aligned}
\Delta_{k} Q_{p}(1 ; n) & =0 & & \text { if } 1 \leqq k \leqq n, k \neq p, \\
& =(n+1) /\left(2 \sin ^{2} p \pi / n+1\right) & & \text { if } k=p,
\end{aligned}
$$

so the polynomials $Q_{p}(z ; n)$ are linearly independent.

THEOREM 2. If $P(z)=z+a_{2} z^{2}+\cdots+a_{n} z^{n} \in \mathscr{P}_{n}$ is such that $a_{n}=1$, then $P(z)$ $=\sum_{p=1}^{n} \alpha_{p} Q_{p}(z ; n)$ where $\alpha_{p}$ is real when $p$ is odd and pure imaginary when $p$ is even. Further $\sum_{p \text { odd }} \alpha_{p}=1$ and $\sum_{p \text { even }} \alpha_{p}=0$.

Proof. Since the $Q_{p}(z ; n)$ are linearly independent, we may write

$$
P(z)=\sum_{p=1}^{n} \alpha_{p} Q_{p}(z ; n) .
$$

Then $\Delta_{p} P(1)=\alpha_{p} \Delta_{p} Q_{p}(1 ; n)$ by $(4)$ and we have $\alpha_{p}=\Delta_{p} P(1)\left(2 \sin ^{2} p \pi /(n+1)\right) /(n+1)$.

As remarked before, $a_{n}=1$ implies the coefficient relation $a_{j+1}=\bar{a}_{n-j}$ when $P \in \mathscr{P}_{n}$ so

$$
\Delta_{p} P(1)=1+\frac{\sin 2 p \pi /(n+1)}{\sin p \pi /(n+1)} a_{2}+\cdots+(-1)^{p-1} \frac{\sin 2 p \pi /(n+1)}{\sin p \pi /(n+1)} \bar{a}_{2}+(-1)^{p-1}
$$

which is real if $p$ is odd and pure imaginary if $p$ is even. The rest of the theorem follows from the normalization of $P$.

From Theorem 1, we easily obtain the following corollary.

CoROLlaRY 1. If $P \in \mathscr{P}_{n}$ is extreme and $a_{n} \geqq 0$ then $P(z)=\sum_{p=1}^{n} \alpha_{p} Q_{p}(z ; n)$ where $\alpha_{p}$ is real when $p$ is odd and pure imaginary when $p$ is even. Further $\sum_{p \text { odd }} \alpha_{p}=1$ and $\sum_{p \text { even }} \alpha_{p}=0$.

In $[6$, p. 496] the polynomials $P(z ; n, j)$ defined by

$$
P(z ; n, j)=\sum_{k=1}^{n} \frac{n-k+1}{n} \frac{\sin k j \pi /(n+1)}{\sin j \pi /(n+1)} z^{k}
$$

were introduced and shown to be univalent. These polynomials are related to the polynomials $Q_{p}(z ; n)$ by the equation $P(z ; n, p)=((n+1) / n) Q_{p}(z ; n)-(1 / n) z Q_{p}^{\prime}(z ; n)$. If $P \in \mathscr{P}_{n}$, let $P^{*}(z)=((n+1) / n) P(z)-(1 / n) z P^{\prime}(z)$. We show below that if $P \in \mathscr{P}_{n}$ and $a_{n}=1$ then $P^{*}$ is univalent in the disk. We require the following lemma.

LEMMA 1. If $P(z)=\sum_{j=1}^{n} a_{j} z^{j} \in \mathscr{P}_{n}$ then

$$
P^{*}(z)=\sum_{j=1}^{n} \frac{n-j+1}{n} a_{j} z^{j} \in \mathscr{P}_{n} .
$$

Proof. Observe that

$$
\Delta_{k} P^{*}(z)=\frac{n+1}{n} \Delta_{k} P(z)-\frac{1}{n} \Delta_{k}\left[z P^{\prime}(z)\right]=\frac{n+1}{n} \Delta_{k} P(z)-\frac{1}{n} z\left(\Delta_{k} P\right)^{\prime}(z) .
$$


Since $\Delta_{k} P(z)=z \prod_{j=1}^{n-1}\left(1-z \mid z_{j}\right),\left|z_{j}\right| \geqq 1$, we have

$$
\operatorname{Re}\left[\frac{z\left(\Delta_{k} P\right)^{\prime}(z)}{\Delta_{k} P(z)}\right]=\operatorname{Re}\left[1-\sum_{j=1}^{n-1} \frac{z / z_{j}}{1-z / z_{j}}\right] \leqq 1+\frac{n-1}{2}=\frac{n+1}{2} .
$$

Hence

$$
\left|\frac{\Delta_{k} P^{*}(z)}{z}\right|=\left|\frac{\Delta_{k} P(z)}{n z}\right|\left|n+1-\frac{z\left(\Delta_{k} P\right)^{\prime}(z)}{\Delta_{k} P(z)}\right| \geqq \frac{\left|\Delta_{k} P(z)\right|}{n|z|} \cdot \frac{n+1}{2} \neq 0
$$

when $|z|<1$.

REMARK. It is also clear in the above proof that $\Delta_{k} P^{*}\left(z_{0}\right)=0$ for some $z_{0}$ on $|z|=1$ if and only if $\Delta_{k} P(z)$ has a double zero at $z=z_{0}$.

THeOREM 3. If $P(z)=\sum_{j=1}^{n} a_{j} z^{j} \in \mathscr{P}_{n}$ and $\left|a_{n}\right|=1$, then $P^{*}$ is univalent in $|z|<1$.

Proof. We may assume $a_{n}=1$. Using the coefficient relation $a_{j+1}=\bar{a}_{n-j}$ and proceeding as in [6, pp. 497-498] we find $e^{i \theta} P^{* \prime}\left(e^{i \theta}\right)=e^{i(n+1) \theta / 2} R(\theta)$ where $R$ is real valued and $\operatorname{Re}\left[e^{i \theta} P^{* \prime \prime}\left(e^{i \theta}\right) / P^{*^{\prime}}\left(e^{i \theta}\right)+1\right]=(n+1) / 2$ when $P^{*^{\prime}}\left(e^{i \theta}\right) \neq 0$. That is, the tangent line to the curve $P^{*}\left(e^{i \theta}\right), 0 \leqq \theta \leqq 2 \pi$, turns at a constant rate in a counterclockwise direction as $\theta$ increases except at the cusps where it reverses direction.

We wish to show that for each $\theta, 0 \leqq \theta<\pi / 2$, the polynomial

$$
S(z, \theta)=\left(P^{*}\left(z e^{i \theta}\right)-P^{*}\left(z e^{-i \theta}\right)\right) / z\left(e^{i \theta}-e^{-i \theta}\right) \quad\left(=P^{* \prime}(z) \text { if } \theta=0\right)
$$

has no zeros in $|z|<1$. We first show $P^{*^{\prime}}(z) \neq 0$ in $|z|<1$ so suppose $S\left(z_{0}, 0\right)=0$ for some $z_{0},\left|z_{0}\right|<1$. Since for each $\theta, S(z, \theta)$ is a polynomial and the zeros of a polynomial vary continuously with the coefficients there is a continuous function $z(\theta)$ such that $S(z(\theta), \theta)=0,0 \leqq \theta \leqq \pi / n+1, z(0)=z_{0}$. By Lemma $1, S(z, \pi /(n+1)) \neq 0$ in $|z|<1$ so $|z(\pi /(n+1))| \geqq 1$. Therefore $|z(\phi)|=1$ for some $\phi, 0<\phi \leqq \pi /(n+1)$. For each $\theta, 0<\theta<\phi$, one can find tangent lines $L(\theta), M(\theta)$ to the closed curve $\gamma(\theta)$ $=\left\{P\left(z(\theta) e^{i \psi}\right):(-\theta \leqq \psi \leqq \theta)\right\}$ such that $\gamma(\theta)$ is contained between $L(\theta)$ and $M(\theta)$ and so that $L$ and $M$ vary continuously with $\theta$ (for example choose $L$ and $M$ parallel to the tangent to $P\left(z(\theta) e^{i \psi}\right)(|\theta-\psi|<\varepsilon)$ where $\varepsilon$ is small). Hence one obtains parallel tangents $L(\phi), M(\phi)$ to the closed curve $\gamma(\phi)$ at the points $P\left(z(\phi) \exp \left(i \psi_{1}\right)\right)$ and $P\left(z(\phi) \exp \left(i \psi_{2}\right)\right)$ where $0<\psi_{2}-\psi_{1}<2 \phi \leqq 2 \pi /(n+1)$. But the tangent line turns at the constant rate $(n+1) / 2$ on $|z|=1$ so $L$ and $M$ parallel implies $\psi_{2}-\psi_{1}$ $=2 k \pi /(n+1) \geqq 2 \pi /(n+1)$ which is a contradiction. We remark that it seems necessary to find $L(\phi)$ and $M(\phi)$ as above to avoid the problem of cusps on the image of $|z|=1$.

Now suppose $S(z, \theta)=0$ for some $\theta$ and $z, 0<\theta<\pi / 2,|z|<1$. Let $r$ be a minimum such that for some $z_{0}$ and $\theta_{0}, r=\left|z_{0}\right|$ and $S\left(z_{0}, \theta_{0}\right)=0$. As before, there is a continuous function $z(\theta), 0 \leqq\left|\theta-\theta_{0}\right| \leqq \pi /(n+1)$, such that $S(z(\theta), \theta)=0$ and $z\left(\theta_{0}\right)=z_{0}$. Again using Lemma 1, we conclude there are $\phi_{1}$ and $\phi_{2}$ such that $-\pi /(n+1)<\phi_{2}$ 
$-\theta_{0}<0<\phi_{1}-\theta_{0}<\pi /(n+1)$ and $\left|z\left(\phi_{1}\right)\right|=\left|z\left(\phi_{2}\right)\right|=1$. If for some continuous branch of the argument, we have

$$
\psi_{1}=\arg \left(z\left(\phi_{1}\right) \exp \left(i \phi_{1}\right)\right)>\arg \left(z\left(\phi_{2}\right) \exp \left(i \phi_{2}\right)\right)=\psi_{2}
$$

and

$$
\psi_{3}=\arg \left(z\left(\phi_{2}\right) \exp \left(-i \phi_{2}\right)\right)>\arg \left(z\left(\phi_{1}\right) \exp \left(-i \phi_{1}\right)\right)=\psi_{4}
$$

we may proceed as in the proof that $P^{* \prime}(z) \neq 0$ in $|z|<1$ to show that there exist $\theta_{1}$ and $\theta_{2}$ satisfying $\psi_{1}>\theta_{1}>\psi_{2}, \psi_{3}>\theta_{2}>\psi_{4}$ and $\theta_{1}-\theta_{2}=2 j \pi /(n+1)>0$. But $\psi_{1}-\psi_{4}$ $=2 \phi_{1}>\theta_{1}-\theta_{2}>\psi_{2}-\psi_{3}=2 \phi_{2}$ so $\phi_{1}>j \pi /(n+1)>\phi_{2}$ and $S(z(j \pi /(n+1)), j \pi /(n+1))$ $=0$ contradicting Lemma 1 .

Let $\gamma$ be the curve $P^{*}\left(z_{0} e^{i \theta}\right), 0 \leqq \theta \leqq 2 \pi$. The curve $\gamma$ has a common tangent line where $\theta=\theta_{0}$ and $\theta=-\theta_{0}$ by the way in which $z_{0}$ and $\theta_{0}$ were chosen. Since $z P^{* \prime}(z)$ is in the direction of the outward normal when $P^{* \prime} \neq 0$ we must have

$$
\frac{z_{0} \exp \left(i \theta_{0}\right) P^{* \prime}\left(z_{0} \exp \left(i \theta_{0}\right)\right)}{z_{0} \exp \left(-i \theta_{0}\right) P^{*^{\prime}}\left(z_{0} \exp \left(-i \theta_{0}\right)\right)}<0 .
$$

Using the fact that $P^{*}\left(z(\theta) e^{i \theta}\right)-P^{*}\left(z(\theta) e^{-i \theta}\right)=0$, we find

$$
\begin{aligned}
\left|\frac{d \arg z(\theta)}{d \theta}\right| & =\left|\operatorname{Im} \frac{d \log z(\theta)}{d \theta}\right| \\
& =\left|\left(1+\frac{z(\theta) e^{i \theta} P^{* \prime}\left(z(\theta) e^{i \theta}\right)}{z(\theta) e^{-i \theta} P^{*^{\prime}}\left(z(\theta) e^{-i \theta}\right)}\right) /\left(1-\frac{z(\theta) e^{i \theta} P^{*^{\prime}}\left(z(\theta) e^{i \theta}\right)}{z(\theta) e^{-i \theta} P^{*^{\prime}}\left(z(\theta) e^{-i \theta}\right)}\right)\right| \\
& <1
\end{aligned}
$$

when $\theta=\theta_{0}$. This means that if $\phi_{1}$ and $\phi_{2}$ are sufficiently near $\theta_{0}$ and such that $\phi_{2}<\theta_{0}<\phi_{1}$ and $\left|z\left(\phi_{1}\right)\right|=\left|z\left(\phi_{2}\right)\right|$ then $\arg \left(z\left(\phi_{2}\right) \exp \left(i \phi_{2}\right)\right)<\arg \left(z\left(\phi_{1}\right) \exp \left(i \phi_{1}\right)\right)$ and $\arg \left(z\left(\phi_{2}\right) \exp \left(-i \phi_{2}\right)\right)>\arg \left(z\left(\phi_{1}\right) \exp \left(-i \phi_{1}\right)\right)$. Therefore to complete the proof of the theorem we need only show that if $1 \geqq\left|z\left(\phi_{2}\right)\right|=\left|z\left(\phi_{1}\right)\right|$ and either

or

$$
\arg \left(z\left(\phi_{2}\right) \exp \left(i \phi_{2}\right)\right)=\arg \left(z\left(\phi_{1}\right) \exp \left(i \phi_{1}\right)\right)
$$

$$
\arg \left(z\left(\phi_{2}\right) \exp \left(-i \phi_{2}\right)\right)=\arg \left(z\left(\phi_{1}\right) \exp \left(-i \phi_{1}\right)\right)
$$

we obtain a contradiction to $P^{*} \in \mathscr{P}_{n}$. We have $0<\phi_{1}-\phi_{2}<\pi /(n+1)$ and by changing notation there are $z$ and $\theta=\phi_{1}-\phi_{2}$ such that $P^{*}\left(z e^{i \theta}\right)=P^{*}\left(z e^{-i \theta}\right)$, $0<\theta<\pi /(n+1)$. The proof now proceeds as in the proof that $P^{*^{\prime}} \neq 0$ in $|z|<1$.

Corollary 2. If $P \in \mathscr{P}_{n}$ is an extreme point then $((n+1) / n) P(z)-(1 / n) z P^{\prime}(z)$ is univalent in $|z|<1$.

We have the following converse to Theorem 3 .

THEOREM 4. If

$$
Q(z)=\sum_{j=1}^{n} \frac{n-j+1}{n} a_{j} z^{j}, \quad a_{1}=1=a_{n},
$$

and $Q(z)$ is univalent in $|z|<1$ then $P(z)=\sum_{j=1}^{n} a_{j} z^{j} \in \mathscr{P}_{n}$. 
Proof. As shown in [1], we must have $a_{\jmath_{+1}}=\bar{a}_{n-j}$ and it then follows that

$$
\operatorname{Re}\left[\Delta_{k} z P^{\prime}(z) / \Delta_{k} P(z)\right]=\operatorname{Re}\left[z\left(\Delta_{k} P\right)^{\prime}(z) / \Delta_{k} P(z)\right]=(n+1) / 2
$$

when $|z|=1, \Delta_{k} P(z) \neq 0$.

Suppose $\Delta_{k} P(z)=0$ for some $z,|z|<1$. Then $w=\Delta_{k} P^{\prime}(z) / \Delta_{k} P(z)=n+1$ for some $z,|z|<1$ for $w$ assumes every value in a neighborhood of $\infty$ and therefore every value not on the line $\operatorname{Re} w=(n+1) / 2$. But $\Delta_{k} Q(z)=((n+1) / n) \Delta_{k} P(z)-(1 / n) \Delta_{k} z P^{\prime}(z)$ $=0$ when $w=n+1$ which contradicts the univalence of $Q$. This proves $\Delta_{k} P(z) \neq 0$ when $|z|<1,1 \leqq k \leqq n$ so $P \in \mathscr{P}_{n}$ and the proof is complete.

Now suppose $P(z)=\sum_{j=1}^{n} a_{j} z^{j} \in \mathscr{P}_{n}$. If $a_{n}=1$, then by Theorem $3, P^{*}$ is univalent in $|z|<1$ so $\left|a_{j}\right|<n(3 j) /(n-j+1)<6 n j /(n+1)<6 j$ if $j \leqq(n+1) / 2$. Using the coefficient relation, $\left|a_{j}\right|<6 j$ for all $j$. If $0 \leqq a_{n}<1$ then as shown in the proof of Theorem $1, P$ is a convex combination of members of $\mathscr{P}_{n}$ having $n$th coefficient \pm 1 . Therefore, in any case $\left|a_{j}\right|<6 j$. Hence for $P \in \mathscr{P}_{n},|P(z)|<6 \sum_{j=1}^{n} j|z|^{j}$ $<6|z| /(1-|z|)^{2}$ so the family $\bigcup_{n=1}^{\infty} \mathscr{P}_{n}$ is locally uniformly bounded and is therefore a normal family.

Theorem 5. Suppose $P_{n_{k}} \in \mathscr{P}_{n_{k}}$ and that $P_{n_{k}} \rightarrow f$ as $k \rightarrow \infty$. Then $f$ is univalent in $|z|<1$.

Proof. Suppose $f$ is not univalent in $|z|<1$. Then there exist $\theta, z$ such that $0<|z|<1,0<\theta<\pi / 2$ and $f\left(z e^{i \theta}\right)=f\left(z e^{-i \theta}\right)$. In fact there exists $r<1$ and a closed interval $I=\left[\theta_{1}, \theta_{2}\right]$ such that the equation $f\left(z e^{i \theta}\right)=f\left(z e^{-i \theta}\right)$ has a solution in $D_{r}=\{z: 0<|z|<r\}$ for each $\theta \in I$. For fixed $\theta \in I$, there exists $k$ such that if $l>k$ then $P_{n_{l}}\left(z e^{i \theta}\right)=P_{n_{l}}\left(z e^{-i \theta}\right)$ has a solution in $D_{(1+r) / 2}$. Let $I_{k}=\left\{\theta \in I: P_{n_{l}}\left(z e^{i \theta}\right)\right.$ $=P_{n_{l}}\left(z e^{-i \theta}\right)$ has a solution in $D_{(1+r) / 2}$ for all $\left.l>k\right\}$. Then $\bigcup_{k=1}^{\infty} I_{k}=I$ so by Baires' theorem [7, p. 76] some $I_{k}$ contains an interval. That is $l>k_{0}$ implies $P_{n_{l}}\left(z e^{i \theta}\right)$ $=P_{n_{l}}\left(z e^{-i \theta}\right)$ has a solution in $D_{(1+r) / 2}$ for all $\theta$ in some fixed interval. This contradicts the definition of $\mathscr{P}_{n}$ and completes the proof.

We now wish to obtain some geometric properties of the univalent polynomial $P^{*}$ associated with extreme points $P \in \mathscr{P}_{n}$. Note that a double zero of $\Delta_{k} P(z)$ is a zero of $\Delta_{k} P^{*}$. Letting $\gamma=\left\{P^{*}\left(e^{i \theta}\right): 0 \leqq \theta<2 \pi\right\}$ we see that a double root of $\Delta_{k} P$ on $|z|=1$ corresponds to a point of self-tangency of $\gamma$ and conversely. Further, we have the following lemma.

Lemma 2. If $P \in \mathscr{P}_{n}$ satisfies $a_{n}=1$ then $\Delta_{k} P$ cannot have a zero of multiplicity greater than 2 .

Proof. Assume $\Delta_{k} P$ has a zero of multiplicity greater than 2 . Then $P^{*}\left(z e^{i k \pi /(n+1)}\right)$ $=P^{*}\left(z e^{-i k \pi /(n+1)}\right)$ and $z e^{i k \pi /(n+1)} P^{* \prime}\left(z e^{i k \pi /(n+1)}\right)=z e^{-i k \pi /(n+1)} P^{* \prime}\left(z e^{-i k \pi /(n+1)}\right)$ for some $z,|z|=1$. If $P^{* \prime}\left(z e^{i k \pi /(n+1)}\right)=0=P^{* \prime}\left(z e^{-i k \pi /(n+1)}\right)$ then it is clear that the images under the mapping $P^{*}$ of small sectors of sufficiently large opening inside the unit circle with vertices at $z e^{i k \pi /(n+1)}$ and $z e^{-i k \pi /(n+1)}$ will overlap contradicting 
the univalence of $Q$. Hence the image of $|z|=1$ has a common tangent at the two points under consideration and as seen previously this implies

$$
\frac{z e^{i k \pi /(n+1)} P^{* \prime}\left(z e^{i k \pi /(n+1)}\right)}{z e^{-i k \pi /(n+1)} P^{* \prime}\left(z e^{-i k \pi /(n+1)}\right)}<0
$$

This is a contradiction which completes the proof.

THEOREM 6. If $P \in \mathscr{P}_{n}(n>2)$ is an extreme point then the curve $\gamma=P^{*}\left(e^{i \theta}\right): 0 \leqq \theta$ $\leqq 2 \pi$ has $n-2$ points of self-tangency. Further, if $P^{*}\left(\exp \left(i \theta_{2}\right)\right)=P^{*}\left(\exp \left(i \theta_{1}\right)\right)$ then $\theta_{2}-\theta_{1}=2 k \pi /(n+1)$ for some integer $k$.

Proof. The last assertion in the theorem follows easily from the fact that $\operatorname{Re}\left[e^{i \theta} P^{* \prime \prime}\left(e^{i \theta}\right) / P^{* \prime}\left(e^{i \theta}\right)+1\right]=(n+1) / 2$ when $P^{* \prime}\left(e^{i \theta}\right) \neq 0$ so the tangent line to $\gamma$ turns at a constant rate between cusps as $\theta$ varies. Since $P^{*}\left(\exp \left(i \theta_{2}\right)\right)=P^{*}\left(\exp \left(i \theta_{1}\right)\right)$ implies there is a common tangent line to $\gamma$ at the above points we must have $((n+1) / 2)\left(\theta_{2}-\theta_{1}\right)=k \pi$ for some integer $k$.

We now proceed to prove the first part of the theorem.

We may assume $P(z)=\sum_{j=1}^{n} a_{j} z^{j}, a_{n}=1$. Note that $\Delta_{k+1} P(z)=-\Delta_{n-k} P(-z)$ so it is sufficient to consider $k \leqq(n+1) / 2$. Further, if $n$ is odd, $\Delta_{(n+1) / 2} P(z)$ is an even function and we may assume for this polynomial that $0 \leqq \arg z<\pi$. Hence we will show that there are $n-2$ values of $z$ such that $\Delta_{k} P(z)$ has a double zero on $|z|=1$ for some $k$ satisfying $1 \leqq k<(n+1) / 2$ or $k=(n+1) / 2$ and $0 \leqq \arg z<\pi$. Suppose this is not the case. We wish to construct

$$
\begin{aligned}
R(z) & =\sum_{n \geqq k>1 ; k \text { odd }} \alpha_{k}\left(Q_{k}(z ; n)-Q_{1}(z ; n)\right)+i \sum_{n \geqq k>2 ; k \text { even }} \beta_{k}\left(Q_{k}(z ; n)-Q_{2}(z ; n)\right) \\
& =\sum_{j=2}^{n-1} b_{j} z^{j}
\end{aligned}
$$

where $\alpha_{k}$ and $\beta_{k}$ are real and $Q_{k}(z ; n)$ is given by (2) so that $P(z)+t R(z) \in \mathscr{P}_{n}$ and $P(z)-t R(z) \in \mathscr{P}_{n}$ for some $t>0, R \not \equiv 0$. Then $P=\frac{1}{2}(P+t R)+\frac{1}{2}(P-t R)$ and $P$ is not extreme ( $R$ must have the above form in order for $P+t R$ and $P-t R$ to satisfy the coefficient relation).

We wish to obtain $n-2$ real linear equations in the $n-2$ unknowns $\alpha_{k}, \beta_{k}$. For each double zero $e^{i \theta}$ of $\Delta_{k} P(z)$ restricted as discussed above, we obtain an equation by setting $i^{k-1} e^{-i(n+1) \theta / 2} \Delta_{k} R\left(e^{i \theta}\right)=0$. The coefficient relation in $Q_{k}(z ; n)$ implies that the $\alpha_{k}$ and $\beta_{k}$ have real coefficients in these equations. Suppose $l$ equations are obtained in this way. The remaining equations are obtained by setting $e^{-i j \pi / 2} R\left(e^{i j \pi /(n+1)}\right)=K, j=1,2, \ldots, n-l-2$, where $K=0$ if the determinant of coefficients is 0 and $K=1$ otherwise. Thus in any case, there is a choice of the $\alpha_{k}$ and $\beta_{k}$ such that $\Delta_{k} R(z)=0$ when $\Delta_{k} P(z)$ has a double zero and $R(z) \not \equiv 0$.

Let $k$ be fixed and consider $S(\theta)=\Delta_{k} P\left(e^{i \theta}\right) / \Delta_{k} R\left(e^{i \theta}\right)$. Suppose $\Delta_{k} P\left(e^{i \theta}\right)$ and $\Delta_{k} R\left(e^{i \theta}\right)$ have $p$ common zeros. Then $S(\theta)$ has $n-1-p$ simple zeros and therefore changes sign at each of these zeros. Hence there exists $t_{k}>0$ such that $S(\theta)$. assumes the values $t_{k}$ and $-t_{k}, n-1-p$ times. This means that all the zeros of 
$\Delta_{k} P(z)+t \Delta_{k} R(z)$ and $\Delta_{k} P(z)-t \Delta_{k} R(z)$ lie on $|z|=1$ when $t \leqq t_{k}$. Setting $t=\min _{1 \leqq k \leqq n} t_{k}$ the proof is now complete.

EXAMPLES. $n=1 . \mathscr{P}_{1}=\{z\}$.

$n=2$. Theorem 2 implies that the extreme points of $\mathscr{P}_{2}$ are rotations of $z+z^{2}$.

$n=3$. Theorem 2 implies that the extreme points of $\mathscr{P}_{3}$ are rotations of polynomials of the form $P(z)=z+a_{2} z^{2}+z^{3}$ where $a_{2}$ is real. Clearly we may assume $a_{2} \geqq 0$. Theorem 6 implies that one of the polynomials $\Delta_{1} P(z)=z+\sqrt{ } 2 a_{2} z^{2}+z^{3}$ or $\Delta_{2} P(z)=z-z^{3}$ has a double zero on $|z|=1$. It follows that $a_{2}=\sqrt{ } 2$ so all extreme points of $\mathscr{P}_{3}$ are rotations of $z+\sqrt{ } 2 z^{2}+z^{3}$.

$n=4$. Theorem 2 implies that the extreme points of $\mathscr{P}_{4}$ are rotations of polynomials of the form $P(z)=z+a_{2} z^{2}+\bar{a}_{2} z^{3}+z^{4}$. Theorem 6 implies that

$$
1+2 a_{2} \cos (\pi / 5) z+2 \bar{a}_{2} \cos (\pi / 5) z^{2}+z^{3}
$$

and

$$
1+2 a_{2} \cos (2 \pi / 5) z-2 \bar{a}_{2} \cos (2 \pi / 5) z^{2}-z^{3}
$$

each have a double zero on $|z|=1$. Applying this to $P^{*}$, each of the polynomials,

$$
\begin{aligned}
& 1+\frac{3}{2} a_{2} \cos (\pi / 5) z+\bar{a}_{2} \cos (\pi / 5) z^{2}+\frac{1}{4} z^{3} \text { and } \\
& 1+\frac{3}{2} a_{2} \cos (2 \pi / 5) z-\bar{a}_{2} \cos (2 \pi / 5) z^{2}-\frac{1}{4} z^{3}
\end{aligned}
$$

has exactly one zero on $|z|=1$. By Cohn's rule [1] and [5, p. 149], if $f(z)=c_{0}+c_{1} z$ $+\cdots+c_{k} z^{k}$ satisfies $\left|c_{0}\right|>\left|c_{k}\right|$ then

$$
f^{*}(z)=\bar{c}_{0} f(z)-c_{k} z^{k} \overline{f(1 / \bar{z})}
$$

has the same zeros as $f$ on $|z|=1$ and the same number of zeros as $f$ in $|z|<1$. Applying Cohn's rule twice to each of the polynomials (5) leads to the linear polynomials

$$
\left(\frac{12 \cos 2 \pi / 5}{\bar{a}_{2}}-\frac{2 \bar{a}_{2}}{a_{2}}\right) z+\frac{36 \cos ^{2} 2 \pi / 5}{\left|a_{2}\right|^{2}}-1
$$

and

$$
\left(\frac{12 \cos \pi / 5}{\bar{a}_{2}}+\frac{2 \bar{a}_{2}}{a_{2}}\right) z+\frac{36 \cos ^{2} \pi / 5}{\left|a_{2}\right|^{2}}-1
$$

(we have used the fact that $\cos 2 \pi / 5=(\sqrt{ } 5-1) / 4$ and $\cos \pi / 5=(\sqrt{ } 5+1) / 4$ so $\cos (2 \pi / 5) \cos \pi / 5=1 / 4)$ each of which has a zero on $|z|=1$. This fact yields the equations

$$
\begin{array}{r}
\left|a_{2}\right|^{4}-16 \operatorname{Re} a_{2}^{3} \cos 2 \pi / 5+72\left|a_{2}\right|^{2} \cos ^{2} 2 \pi / 5-432 \cos ^{4} 2 \pi / 5=0, \\
\left|a_{2}\right|^{4}+16 \operatorname{Re} a_{2}^{3} \cos \pi / 5+72\left|a_{2}\right|^{2} \cos ^{2} \pi / 5-432 \cos ^{4} \pi / 5=0 .
\end{array}
$$

Eliminating $\operatorname{Re} a_{2}^{3}$ from the equations (6) we obtain $\left|a_{2}\right|^{4}+18\left|a_{2}\right|^{2}-54=0$ so $\left|a_{2}\right|^{2}$ $=3 \sqrt{ } 15-9$. Substitution into either equation in (6) then yields

$$
\cos \left(3 \arg a_{2}\right)=\frac{3}{16} \sqrt{ }(9+5 \sqrt{ } 15) \text {. }
$$


If we choose a value of $\arg a_{2}$ to satisfy (7) and choose $\left|a_{2}\right|$ so the equations (6) are satisfied then the extreme points in $\mathscr{P}_{4}$ are rotations of the polynomials $z+a_{2} z^{2}$ $+\bar{a}_{2} z^{3}+z^{4}$ and $z+\bar{a}_{2} z^{2}+a_{2} z^{3}+z^{4}$.

$n=5$. The extreme points of $\mathscr{P}_{5}$ are rotations of polynomials of the form $P(z)$ $=z+\left(a+b_{i}\right) z^{2}+c z^{3}+\left(a-b_{i}\right) z^{4}+z^{5}$ where $a, b$ and $c$ are real. By considering $-i P(i z),-P(-z)$ and $(P(\bar{z}))^{-}$we see that we may assume $a, b$ and $c$ are nonnegative. Theorem 6 implies that among the roots of the equations

$$
\begin{aligned}
& 1+\sqrt{ } 3\left(a+b_{i}\right) z+2 c z^{2}+\sqrt{ } 3\left(a-b_{i}\right) z^{3}+z^{4}=0 \\
& 1+\left(a+b_{i}\right) z \quad-\left(a-b_{i}\right) z^{3} \quad-z^{4}=0 \\
& 1-c z^{2} \quad+z^{4}=0
\end{aligned}
$$

there must be three double roots on $|z|=1$ (only half of the double roots of the third equation are to be counted). Observe that $\left(1+e^{i \alpha} z\right)^{2}\left(1+e^{i \beta} z\right)^{2}=1+2\left(e^{i \alpha}+e^{i \beta}\right) z$ $+\left(e^{2 i \alpha}+e^{2 i \beta}+4 e^{i(\alpha+\beta)}\right) z^{2}+2\left(e^{i(2 \alpha+\beta)}+e^{i(2 \beta+\alpha)}\right) z^{3}+e^{i 2(\alpha+\beta)} z^{4}$ so the second equation in (8) cannot have two double roots on $|z|=1$. Suppose the first equation has two double roots on $|z|=1$. Then the left hand-side has the form above where $\beta=-\alpha$ or $\beta=\pi-\alpha$. Since $c \geqq 0$, we must have $\beta=-\alpha$ so $b=0$. The second equation in (8) then has roots \pm 1 together with the zeros of $1+a z+z^{2}$. Hence in this case the second equation cannot have a double root (the only possibility is -1 and it is either a simple root or a triple root). This means the third equation has a double root so $c=2$ and $a=\sqrt{ } \frac{8}{3}$. But

$$
z+\sqrt{ } \frac{8}{3} z^{2}+2 z^{3}+\sqrt{ } \frac{8}{3} z^{4}+z^{5}=((\sqrt{ } 8+3) / 6) Q_{1}(z ; 5)+((3-\sqrt{ } 8) / 6) Q_{5}(z ; 5)
$$

and this polynomial is not an extreme point.

Thus we conclude that if $P$ is extreme then each of the equations in (8) has a double root on $|z|=1$. From the third equation, $c=2$. Assume that $e^{i \phi}$ and $e^{i \theta}$ are double roots of the first and second equations respectively. We obtain the system

$$
\begin{aligned}
\cos 2 \phi+\sqrt{ } 3 a \cos \phi+\sqrt{ } 3 b \sin \phi+2 & =0 \\
2 \sin 2 \phi+\sqrt{ } 3 a \sin \phi-\sqrt{ } 3 b \cos \phi & =0 \\
\sin 2 \theta+a \sin \theta-b \cos \theta & =0 \\
2 \cos 2 \theta+a \cos \theta+b \sin \theta & =0
\end{aligned}
$$

Solving for $a$ and $b$ in terms of $\theta$ and $\phi$ we find

$$
\begin{aligned}
& a=-2 \cos ^{3} \theta=(1 / 2 \sqrt{ } 3)(\cos 3 \phi-7 \cos \phi), \\
& b=2 \sin ^{3} \theta=(1 / 2 \sqrt{ } 3)(\sin 3 \phi-\sin \phi) .
\end{aligned}
$$

From equations (10),

$$
a^{2}+b^{2}=\frac{1}{3}\left(8+4 \cos 2 \phi-3 \cos ^{2} 2 \phi\right)
$$

so $a^{2}+b^{2}<28 / 9$.

Now let $\theta=\theta(\phi)$ satisfy $-2 \cos ^{3} \theta=(1 / 2 \sqrt{ } 3)(\cos 3 \phi-7 \cos \phi)$ and set $g(\phi)$ $=2 \sin ^{3} \theta-(1 / 2 \sqrt{ } 3)(\sin 3 \phi-\sin \phi)$. Since $a>0$ and $b>0$ we have $\pi>\phi, \theta>3 \pi / 4$. 
Also $g^{\prime}(\phi)=\left(10-12 \cos ^{2} \phi\right) \cos (\phi-\theta) /(2 \sqrt{ } 3 \cos \theta), g(\pi)>0, g\left(\phi_{0}\right)<0$ and $g(3 \pi / 4)$ $>0$ where $\cos ^{2} \phi_{0}=\frac{5}{6}$. Thus we conclude the system (10) has two solutions. Assume the values of $\phi$ corresponding to these solutions are $\phi_{1}$ and $\phi_{2}$ where $\pi>\phi_{1}>\phi_{0}>\phi_{2}$ $>3 \pi / 4$. From (11), we find $a^{2}+b^{2}=3$ when $\cos 2 \phi=1, \frac{1}{3}$ while $g(\phi)<0$ when $\cos 2 \phi=\frac{1}{3}$ so $3<a^{2}+b^{2}<28 / 9$ for the solution corresponding to $\phi_{1}$ and $a^{2}+b^{2}<3$ for the solution corresponding to $\phi_{2}$. Denote the polynomials $P$ corresponding to the solutions of (10) for $\phi=\phi_{1}, \phi_{2}$ by $P_{1}$ and $P_{2}$ respectively and let $\theta_{1}$ and $\theta_{2}$ be the corresponding values of $\theta$. Clearly $P_{1}$ is an extreme point since it maximizes $\left|a_{2}\right|$ $\left(P_{1} \in \mathscr{P}_{5}\right.$ since there must be an extreme point satisfying $\left.\sqrt{ } 3 \leqq\left|a_{2}\right|\right)$.

We now show $P_{2}$ is also an extreme point of $\mathscr{P}_{5}$. Note that in any of the equations in (8), the roots which do not lie on $|z|=1$ must occur as pairs of roots which are inverse points with respect to $|z|=1$. Hence if two roots vary continuously beginning on $|z|=1$ and ending as inverse points with respect to the circle then they must at some time coincide. The third equation in (8) has its roots on the circle when $c=2$. Setting $a(\theta)=-2 \cos ^{3} \theta$ and $b(\theta)=2 \sin ^{3} \theta, e^{i \theta}$ is a double root of the second equation and the other roots lie on $|z|=1$ for we can never have $a(\alpha)=a(\theta)$ and $b(\alpha)=b(\theta)$ when $\alpha \neq \theta \bmod 2 \pi$ (and the roots do lie on $|z|=1$ when $\theta=0$ ). Now set $a(\phi)=(1 / 2 \sqrt{ } 3)(\cos 3 \phi-7 \cos \phi)$ and $b(\phi)=(1 / 2 \sqrt{ } 3)(\sin 3 \phi-\sin \phi)$. All roots of the first equation in (8) lie on $|z|=1$ when $\phi=\pi$. Further, if $\pi>\phi>3 \pi / 4$ then $a(\phi)=a(\alpha)$ and $b(\phi)=b(\alpha)$ together imply $\phi=\alpha$. Hence for $\phi=\phi_{2}$, all roots of the first equation in (8) lie on $|z|=1$. Hence $P_{2} \in \mathscr{P}_{5}$.

Now suppose $P_{2}$ is not an extreme point. Then $P_{2}$ is in the convex hull of the set

$$
\left\{e^{-i \alpha} P_{1}\left(e^{i \alpha} z\right): \alpha \text { is real }\right\} \cup\left\{e^{i \beta} \overline{P_{1}\left(\bar{z} e^{i \beta}\right)}: \beta \text { is real }\right\} .
$$

Since $P_{2}$ has third coefficient 2 and fifth coefficient $1, P_{2}$ is a convex combination of

$$
P_{1}(z), \quad \overline{P_{1}(\bar{z})}, \quad-P_{1}(-z) \text { and } \overline{-P_{1}(-\bar{z})} \text {. }
$$

Therefore, $\sin ^{3} \theta_{2}$ is a convex combination of $\sin ^{3} \theta_{1}$ and $-\sin ^{3} \theta_{1}$. But $\sin \theta_{2}$ $>\sin \theta_{1}$ and this is impossible so $P_{2}$ is extreme.

For the next theorem, we restrict ourselves to the subclass $\mathscr{R}_{n} \subset \mathscr{P}_{n}$ having the property $P \in \mathscr{R}_{n}$ implies $P$ has real coefficients.

THEOREM 7. For each $p=1,2, \ldots, n, Q_{p}(z ; n)$ is an extreme point of $\mathscr{R}_{n}$. Further $\left.\mathscr{R}_{n} \subset \operatorname{co}\left\{Q_{p}(z ; n)\right\}_{p=1}^{n}\right)$ where co $(A)$ is the convex hull of $A$.

Proof. Let $P(z)=z+\sum_{j=2}^{n} a_{j} z^{j} \in \mathscr{R}_{n}$ be extreme. As in the proof of Theorem 1, we may show that if $P$ is extreme then $a_{n}= \pm 1$. Using Theorem 3 above, Theorem 2 of $\left[6\right.$, p. 500] and the fact mentioned previously that $Q_{p}^{*}(z ; n)=P(z ; n, p)$ we have $P(z)=\sum_{p=1}^{n} \alpha_{p} Q_{p}(z ; n)$ where $\alpha_{p} \geqq 0$ and $\sum_{p=1}^{n} \alpha_{p}=1$. This proves

$$
\mathscr{R}_{n} \subset \operatorname{co}\left(\left\{Q_{p}(z ; n)\right\}_{p=1}^{n}\right) \text {. }
$$

Every $Q \in \mathscr{R}_{n}$ can be written uniquely in the form $Q(z)=\sum_{p=1}^{n} \alpha_{p} Q_{p}(z ; n), \alpha_{p} \geqq 0$, $\sum_{p=1}^{n} \alpha_{p}=1$ and the functional $J_{k}$ defined on $\mathscr{R}_{n}$ by $J_{k}(Q)=\alpha_{k}$ is a continuous linear 
functional. Clearly $\alpha_{k}=1$ is its maximum which is assumed only when $Q(z)=Q_{k}(z ; n)$. This proves $Q_{k}(z ; n)$ is extreme in $\mathscr{R}_{n}$.

From Theorems 6 and 7 above, it seems likely that no extreme points of $\mathscr{P}_{n}$ can have all real coefficients when $n \geqq 4$. At least we have the following theorem.

THEOREM 8. If $n>5$ and $1<j<n$ then

$$
\max _{P \in \mathscr{P}_{n}}\left|a_{j}\right|>\frac{\sin j \pi /(n+1)}{\sin \pi /(n+1)}=\max _{P \in \mathscr{R}_{n}}\left|a_{j}\right|
$$

where $P(z)=\sum_{j=1}^{n} a_{j} z^{j}$.

Proof. The equality

$$
\max _{P \in \mathscr{R}_{n}}\left|a_{j}\right|=\frac{\sin j \pi /(n+1)}{\sin \pi /(n+1)}
$$

follows from Theorem 3 and [6, Theorem 3]. From (3) we see that $\Delta_{k} Q_{1}(z ; n) / z$ has simple zeros except possibly at \pm 1 and $\Delta_{k}\left[Q_{4}(z ; n)-Q_{2}(z ; n)\right] / z$ has a simple zero at \pm 1 when $\Delta_{k} Q_{1}(z ; n) / z$ has a double zero, $n>5$. It then follows by the same argument as used in the proof of Theorem 6 that for $t$ sufficiently small, $Q(z)$ $=Q_{1}(z ; n)+i t\left[Q_{4}(z ; n)-Q_{2}(z ; n)\right] \in \mathscr{P}_{n}$ and all zeros of $\Delta_{k} Q$ are simple zeros, $1 \leqq k \leqq n$. For fixed $j$, choose $p$ odd so that

$$
\left|\frac{\sin j p \pi /(n+1)}{\sin p \pi /(n+1)}\right|<\frac{\sin j \pi /(n+1)}{\sin \pi /(n+1)} .
$$

Applying the same argument, it follows that, for sufficiently small s,

$$
P(z)=Q_{1}(z ; n)-s\left(Q_{p}(z ; n)-Q_{1}(z ; n)\right)+i t\left(Q_{4}(z ; n)-Q_{2}(z ; n)\right)
$$

is in $\mathscr{P}_{n}$. Then $\left|a_{j}\right| \geqq \operatorname{Re} a_{j}>(\sin \mathrm{j} \pi /(n+1)) /(\sin \pi /(n+1))$. Actually the conclusion of the theorem holds for $n>3$ except for the case $n=5, j=3$.

THEOREM 9. Every function $f$ in the class $S$ of functions univalent in $|z|<1$ and normalized by setting $f(0)=0, f^{\prime}(0)=1$, is the limit of polynomials of the form $P(z)$ $=z+\sum_{j=2}^{n} a_{j} z^{j} \in \mathscr{P}_{n}$ which satisfy $a_{n}=1$.

Proof. Let $f \in S$. One can obtain a sequence of univalent polynomials by taking appropriate partial sums of $r_{k}^{-1} f\left(r_{k} z\right)$ where $\left\{r_{k}\right\}_{k=1}^{\infty}$ is a strictly increasing sequence of real numbers such that $\lim _{k \rightarrow \infty} r_{k}=1$. Let $\left\{Q_{n_{k}}\right\}$ be such a sequence where $Q_{n_{k}}$ is of degree $n_{k}$. Define

$$
P_{n_{k}}(z)=Q_{n_{k}}(z)+z^{2 n_{k}+1} \overline{Q_{n_{k}}(1 / z)}=\sum_{j=1}^{2 n_{k}} a_{j} z^{j} .
$$

By an argument similar to that used to prove Theorem 1, $P_{n_{k}} \in \mathscr{P}_{2 n_{k}}$ and $a_{2 n_{k}}=1$. Also $\lim _{k \rightarrow \infty} P_{n_{k}}(z)=f(z)$ so $\left\{P_{n_{k}}\right\}_{k=1}^{\infty}$ is the required sequence. 
THEOREM 10. The univalent polynomials of the form $z+\sum_{j=2}^{n} a_{j} z^{j}$ which satisfy $(j+1) a_{j+1}=(n-j) \bar{a}_{n-j}\left[\right.$ and thus $\left.a_{n}=1 / n\right]$ are dense in the class $S$.

Proof. The polynomials $\left\{P_{n_{k}}^{*}\right\}_{k=1}^{\infty}$ have the same limit as $\left\{P_{n_{k}}\right\}_{k=1}^{\infty}$ above.

We observe that in Theorem 9 , if $f \in S$ has real coefficients then the sequence $\left\{P_{n_{k}}^{*}\right\}_{k=1}^{\infty}$ has real coefficients and we have a new proof of the Bieberbach conjecture for functions having real coefficients.

Let $\mathscr{D}_{n}$ be the class of polynomials of degree $n$ which are univalent in $|z|<1$ and of the form $z+\sum_{j=2}^{n} a_{j} z^{j},(j+1) a_{j+1}=(n-j) \bar{a}_{n-j}$. Applying Theorems 2 and 4 above and using the definition of $P(z ; n, j)$ in [6] we can represent any $P \in \mathscr{D}_{n}$ in the form

$$
\begin{aligned}
P(z)= & P(z ; n, 1)+\sum_{j \text { odd }} \alpha_{j}[P(z ; n, j)-P(z ; n, 1)] \\
& +i \sum_{j \text { even }} \beta_{j}[P(z ; n, j)-P(z ; n, 2)] .
\end{aligned}
$$

Since the tangent line to the curve $P\left(e^{i \theta}\right)(0 \leqq \theta \leqq 2 \pi)$ turns at a constant rate $\left(\operatorname{Re}\left[e^{i \theta} P^{\prime \prime}\left(e^{i \theta}\right) / P^{\prime}\left(e^{i \theta}\right)+1\right]=(n+1) / 2\right.$ when $\left.P^{\prime}\left(e^{i \theta}\right) \neq 0\right)$ as $\theta$ varies the tangent line to the curve is horizontal when $\theta$ is an odd multiple of $\pi /(n+1)$ and vertical when $\theta$ is an even multiple of $\pi /(n+1)$. Recall that among all polynomials in $\mathscr{D}_{n}$ having real coefficients, $P(z ; n, 1)$ maximizes every coefficient. Also $\Delta_{k} P(1 ; n, 1)=0$ when $k$ is odd, $k>1$ (i.e. $P\left(e^{i k \pi /(n+1)} ; n, 1\right)=P\left(e^{-i k \pi /(n+1)} ; n, 1\right)$ when $k$ is odd, $\left.k>1\right)$. Note also that for even $j>2$ and odd $k, \Delta_{k}[P(1 ; n, j)-P(1 ; n, 2)]$ is pure imaginary. Hence the effect of adding it $[P(z ; n, j)-P(z ; n, 2)]$ (where $t$ is real and near 0 and $j$ is even, $j>2)$ to $P(z ; n, 1)$ is to shift the values at $e^{i k \pi /(n+1)}$ and $e^{-i k \pi /(n+1)}$ apart horizontally. Finally, in the representation (12), for odd $k>1$ we have $\alpha_{k} \operatorname{Im}\left[\Delta_{k} P(1 ; n, k)\right]=\operatorname{Im}\left[\Delta_{k} P(1)\right] \quad$ so $\alpha_{k}$ is negative when $\operatorname{Im} P\left(e^{-i k \pi /(n+1)}\right)$ $>\operatorname{Im} P\left(e^{i k \pi /(n+1)}\right)$. Since the coefficients in $P(z ; n, k)-P(z ; n, 1)$ are all nonpositive, it would appear that to obtain the maximum modulus for any coefficient, one should choose the $\beta_{j}$ to shift the values $P\left(e^{i k \pi /(n+1)}\right)$ and $P\left(e^{-i k \pi /(n+1)}\right)$ apart and then choose the $\alpha_{j}$ negative, the choices being made to satisfy Theorem 6 . This discussion leads to the following conjecture.

Conjecture. Among all polynomials $P(z)=z+\sum_{j=2}^{n} a_{j} z^{j} \in \mathscr{D}_{n}$, the quantities $\left|a_{j}\right|, 2 \leqq j \leqq n-1$, are all maximized by a single polynomial having the property that in the representation (12), $\alpha_{j} \leqq 0$ for all odd $j, n \geqq j>1$.

This conjecture implies the Bieberbach conjecture as shown by the following argument. Suppose $P_{n}(z)$ maximizes $\left|a_{j}\right|, 2 \leqq j \leqq n-1$, in $\mathscr{D}_{n}$. Let $f(z)=\sum_{j=1}^{\infty} b_{j} z^{j} \in S$ and let $Q_{n_{k}} \in \mathscr{D}_{n_{k}}$ where $\left\{Q_{n_{k}}\right\}_{k=1}^{\infty}$ is a sequence of polynomials having $f$ as limit. Let $P_{n}(z)=\sum_{j=1}^{n} a_{j, n} z^{j}, Q_{n_{k}}(z)=\sum_{j=1}^{n_{k}} b_{j, n_{k}} z^{j}$. Then $\left|a_{j, n_{k}}\right| \geqq\left|b_{j, n_{k}}\right|$ for each $k$ and $2>\left|a_{2, n_{k}}\right| \geqq\left(\left(n_{k}-1\right) / n_{k}\right) \cos \pi /\left(n_{k}+1\right)$. Hence $\lim _{k \rightarrow \infty}\left|a_{2, n_{k}}\right|=2$ and any convergent subsequence of $\left\{P_{n}\right\}$ must converge to a Koebe function. After possibly renaming to obtain a convergent subsequence, we therefore have

$$
j=\lim _{k \rightarrow \infty}\left|a_{j, n_{k}}\right| \geqq \lim _{k \rightarrow \infty}\left|b_{j, n_{k}}\right|=\left|b_{j}\right| .
$$




\section{REFERENCES}

1. D. A. Brannan, Coefficient regions for univalent polynomials of small degree, Mathematika 14 (1967), 165-169. MR 36 \#3971.

2. Louis Brickman, Extreme points of the set of univalent functions, Bull. Amer. Math. Soc. 76 (1970), 372-374. MR 41 \#448.

3. J. Dieudonné, Recherches sur quelques problèmes relatifs aux polynomes et aux fonctions bornées d'une variable complexe, Ann. Ecole Norm. Sup. (3) 48 (1931), 247-358.

4. N. Dunford and J. T. Schwartz, Linear operators. I: General theory, Pure and Appl. Math., vol. 7, Interscience, New York, 1958. MR 22 \#8302.

5. M. Marden, The geometry of the zeros of a polynomial in a complex variable, Math. Surveys, no. 3, Amer. Math. Soc., Providence, R. I., 1949. MR 11, 101.

6. T. J. Suffridge, On univalent polynomials, J. London Math. Soc. 44 (1969), 496-504. MR 38 \#3419.

7. A. E. Taylor, Introduction to functional analysis, Wiley, New York, 1958. MR 20 \#5411.

Department of Mathematics, University of Kentucky, LeXington, Kentucky 40506 